\title{
Téoros
}

Revue de recherche en tourisme

\section{Les Laurentides du Nord de Montréal et d'Ottawa : un aperçu de l'organisation spatiale}

\section{Claude Lamothe}

Volume 15, numéro 1, printemps 1996

Les Laurentides, quelles Laurentides?

URI : https://id.erudit.org/iderudit/1075045ar

DOI : https://doi.org/10.7202/1075045ar

Aller au sommaire du numéro

Éditeur(s)

Université du Québec à Montréal

ISSN

0712-8657 (imprimé)

1923-2705 (numérique)

Découvrir la revue

Citer cet article

Lamothe, C. (1996). Les Laurentides du Nord de Montréal et d'Ottawa : un aperçu de l'organisation spatiale. Téoros, 15(1), 4-7.

https://doi.org/10.7202/1075045ar d'utilisation que vous pouvez consulter en ligne.

https://apropos.erudit.org/fr/usagers/politique-dutilisation/ 


\section{Claude Lamothe \\ est géographe-aménagiste. \\ II est président de la Société \\ technique d'aménagement régional inc. (Sotar).}

\section{Une portion du Bouclier canadien}

Les Laurentides du nord de Montréal et d'Ottawa constituent une portion de la bordure méridionale du Bouclier canadien, plateau montagneux qul s'étend au Québec et en Ontario entre les basses terres du SaintLaurent et des Grands Lacs d'une part, et les basses terres de la balc James el la bale d'Hudson d'autre part.

Les Laurentides du nord de Montréal et d'Ottawa dénomment l'ensemble du Bouclier canadien compris entre le versant est de la vallée de la Nolre et le versant ouest de la vallée de la Gatineau; la llmite nord de ces Laurentides est un axe qui passe au nord des réservolrs Baskatong et Taureau, tandis que la limite sud passe par Hull, Lachute, Saint-Jérôme et Saint-Gabriel-de-Brandon. La démarcation entre le plateau laurentidlen et les basses terres laurentiennes est très nette à l'ouest de Lachute puisque le front des Laurentides est sl= gnalé par des abrupts et des escarpements importants, alors que plus à l'est, vers Lanaudière, cette démarcation est plus estompée, car les Laurentides y sont précédées d'un pledmont de petites collines à pentes douces.

\section{Le toponyme Laurentides}

Ces Laurentides font partie des réglons touristiques de l'Outaouais, des Laurentides et de Lanaudière et constituent. en quelque sorte los Laurentides dites touristiques. Cependant, les Laurentides dites géographiques sont beaucoup plus vastes car elles s'étendent à l'est. jusqu'au Saguenay, et à l'ouest jusqu'à l'Outaouals supérieur et à la frontiere de l'Ontario. Le toponyme Laurentides est toutefois rarement utillsé pour référer aux autres portıons du plateau laurentidien situé à l'est de Lanaudlère et à l'ouest de la Gatineau. Au nord de Trols-Rivlères, le plateau laurentidien est plus connu comme les collines de la Mauricle, alors que, plus à l'est, on réfêre davantage aux collines de Portneuf et aux montagnes de Charlevolx pour identifier les autres portions du système laurentidien, malgré le falt que le massil montagneux le plus important au nord de Québec solt situé au coeur d'un territolre Identifié comme la réserve faunique des Laurentides.

En falt. les collines du Boucller canadien auxquelles on accole spontanément le toponyme Laurentides sont celles du nord de Montréal, dans la mesure où les Laurentides du nord d'Ottawa sont dénommés par les anglophones The Gatineau Hills et que leur massif montagneux le plus important est davantage connu sous le nom du Parc de la Gatineau.
Cependant, les Laurentides du nord de Montréal et d'Ottawa ont en commun d'être le terrain de jeux et de villéglature de Montréal et d'Otawa-Hull et de posséder des Installations récréotouristiques variées qul attirent visiteurs et vacanclers.

Par contre, une grande partle des Laurentides du nord de Montréal ct d'Ottawa reste méconnue du public québécols puisque la majorité des visiteurs y clrculent sur les quatre axes principaux nord-sud qul permettent d'accéder au terrltoire, solt à l'est, les autoroutes 25 et 31 et leur prolongement que sont les routes 125 et 131, au centre, l'autoroute 15 et son prolongement la route 117 , à l'ouest l'autoroute 5 et son prolongement la route 105 . Labsence de liens routiers est-ouest majeurs à travers les Laurentides explique sans doute pourquol la plupart des visiteurs ont une perception du territoire limitée à celle qul leur est offerte en clrculant sur les nationales $117,105,131$ ou 125 .

Pourtant les Laurentides ont beaucoup d"autres paysages à offrir que celul du corridor Saint-Jérôme / Salnt-Jovlte et celui du corridor Rawdon / Saint-Donat en ce qul concerne la population montréalalse. ou celul du corrldor Chelsea / Maniwaki en ce qui concerne la population de l'agglomération Hull-Ottawa. En référant à la carte cl-jolnte de l'organlsation spatiale des Laurentides, telle qu'exprimée par l'utilisation et l'aflectation du sol, le découpage géographique et territorial ct le mode de mise en valeur, on peut constater que les espaces affectés à la villégiature, certes, occupent une place importante, mais qu'il en est de même des espaces consacrés à la foresterio, à l'agriculture ou à la conservation.

En falt les Laurentides du nord de Montréal et de HullOttawa ont l'allure d'un immense patchwork qu'on peut decrire de la façon suivante:

1 Les espaces de villéglature et de récréotourisme structurés par une route natlonale ou réglonale d'accès aux Laurentides

Ce sont:

1a) le couloir de la rivlère du Nord, de Saint-Jérôme à SaintFaustin, Incluant la vallée de Saint-Sauveur; la route 117

1b) le couloir aval de la Rouge, de Huberdeau à Labelle; les routes 117 et 327 :

1c) le couloir amont de la Rouge, de Labelle à l'Ascension; la route 117:

1d) lc couloir amont de la Ouareau et plus particullèrement la cuvelte de Saint=Donat avec les lacs Archambault et Ouareau; la route 125 ; 
1e) le haut pays de Paplneau, de Mayo à la tete du lac Gagnon; les routes 315 et 321 ;

Ces cinq entités spatiales sont toutes structurées par une route nationale ou réglonale qul permet d'accéder aux Laurentides; on y retrouve des villes et villages relativement blen étoffés en commerces de blens et de services, des Installations récréotouristiques et de l'hébergement commerclal (chalets de locatlon, hôtels-motels, terrains de camplng. etc.). Ce sont les cinq entités spatiales qui correspondent le plus a l'image de terraln récréatif et ludique qu'ont les Québécols et les Ontariens des Laurentides. On y retrouve la maleure partle des Installations récréotouristiques, des restaurants, des hôtels et des motels des Laurentides alns! que les villages les plus connus.

\section{2' Les espaces de vlliéglature de la bordure méridionale des Iaurentldes}

Ce sont:

2a) le plateau de Lanaudlère, de Salnt-Callxte ă Salnt-Jeande-Matha;

2b) le plateau d'Argenteull, de Bellefeullle à Saint-Mlchel-deWentworth:

2c) I"Interfluve Inférleur Llèvre-Gatineau, de Val-des-Monts à Poltimore:

2d) l'arrlère-pays de Pontlac, solt la bordure des Laurentides au nord-ouest du Parc de la Gatineau.

Cos quatre entités spatiales partagent un même dénominateur commun, à savoir leur proximité́ des contres de populaUlon des basses terres du Salnt-Laurent et de l'Outaouals, la multitude des petits lacs et autres plans d'eau, l'importance de leur réseau routler local, relatwement blen rellé au réseau de voirle nationale. Ces espaces sont surtout utilisés par une cllentèle dhablués, propritetaires de résidences secondalres et de chalets: Ics quelques installations récréotouristiques qu'on y trouve sont surtout conçes pour desservir cette clientèle captive de villéglateurs dont un bon nombre fréquentent la réglon à l'année longue. En plusleurs endrolts, l'appropriation du territolre date du début des années 1930, notamment dans Argenteull, au nord de Brownsburg. Ces espaces de la bordure méridionale des Laurentldes, alnsl que le coulolr de la Nord, jusqu'à SalnteAdèle et celul de la Gatuneau lusqu'à Wakefleld, sont devenus dans les quinze dernlères années des exlensions des banlleues du nord de Montréal et d'Ottawa.

\section{3" Les espaces dont l'appropriation du sol est parlagće entre la villéglature et l'agriculture}

Cesont:

3a) les contreforts de Lanaudiere, solt la bordure des Laurentides comprise entre les rivières LAssomptlon ot Maskinonge:

3b) le coulolr de la Haute Liève, de Notre-Dame-du-Laus à Sainte-Anne-du-Lac;

3c) les bouches des rivleres Petite-Nation. Petite-Rouge ol Blanche:

3d) le coulolr de la Gatineau, de Chelsea à Grand-Remous.
Ces quatre entités spatiales des Laurentides conservent encore leur caractère agricole ou de colonlsation, blen que la villéglature y accapare les rives de leurs cours d'eau et de leurs lacs. Les dépôts limono-argileux, qui tapissent les fonds des vallées et la partie inférleure de leurs versants, nous rappellent que la mer de Champlain a pénétré profondément dans les Laurentides et y a favorlsó le développement d'une agriculture plus prospère qui persiste encore de nos jours. Lá Lol de la protection des terres agricoles a empêché jusqu'à ce jour leur approprlation à des fins autres qu'agricoles, ce qul contribue à maintenlr la villéglature et le récréotourisme dans des espaces conflnćs et relativement restreints.

4" Les espaces aux paysages agralres remarquables et peu touchés par la viléglature et l'urbanisation

Ce sont:

4a) les bouches de la Rouge, de Polnte-au-Chëne et Calumet |usqu"à Bróbeuf;

4b) le coulolf aval de la Lievre, de Buckingham à Val-desBols.

Ces deux entités spatlales sont en quelque sorte des enclaves agricoles insérés dans les Laurentides. Le coulolr de la Llève est une des plus belles unités de paysage des Laurentides du nord de Hull-otlawa par ses champs et pâturages ćtalés sur les ondulations du fond de la vallée mals aussl sur les versants à pente douce où lls partagent l'espace avec des bolsés de ferme. Bien que le coulolr de la Lière ature davantage de vislteurs et de villéglateurs depuls la mise en valeur par la Soclété d'Aménagement de l'Outaouals, dans les années 1975, du complexe touristique du lac de l'Argile (maintenant géré par la Soclété des établissements de plein alr du Québec). le caractère rural et champettre orlginal a pu être conservé, notamment à cause de la faible utilisation de la route 309 comme vole de pénetration des Hautes-Laurentides.

Les bouches de la Rouge sont pour leur part un coln des Laurentides oủ l'assoclation paysage agralre, paysage forestior est des plus harmonleuse. Entre Brébeuf et Huberdeau-Arundel, la valléc aval de la Rouge se présente comme une très belle auge glaclaire dont les rives et les versants sont appropriés par des champs et des pãturages, lui conférant un caractère champêtre assez unlque dans les Laurentıdes. Au sud d'Arundel, la rlvlère Rouge devlent encalssce et a même des allures d'une gorge entallée à travers les montagnes. Elle est accessible uniquement par le róseau routier local; l'agriculture y est marginale, mals les champs et les paturages défrichés par les plonnters anglosaxons sont encore conservés et contribuent à créer à travers ce dédale de collines abruptes des petits ensembles champêtres d'une beauté remarquable et parsemés de lieuxdits Gvocateurs comme Gray Valley. Rockway Valley. Rivington, Harrington, etc. Exception faite des amateurs de rafting. peu de Québécols connalssent ce coln enchanteur dos Laurentides.

\section{Les espaces de grande nature Intégrés majorltalre- ment au domaine public et axés sur I'exploitation contrôléce de la faune et de la forêt}


Ce sont:

5a) le domaine public de Mastlgouche, soit essentiellement la réserve faunlque Mastligouche et la ZEC des Nymphes:

5b) le domaine public de la Noire-LAssomption. approprié en grande partle par la ZEC Lavigne;

5c) le domaine public de la Rouge-Mattawin, solt essentlellement la réserve faunique Rouge-Mattawin et la ZEC Collin:

5d) le domalne public de la Rouge-Lièvre, solt le territoire entourant le réservolr Klamlka Incluant la ZEC Malsonde-plerre:

5e) le domaine public Paplneau-Labelle, solt essentlellement la réserve faunlque du même nom, ainsl que des terres publlques adjacentes à la réserve:

5f) le domaine public de la rlvière Michel, solt le plateau montagneux de la têtte des eaux du bassin de la Ouareau au sud du massif du parc du Mont-Tremblant;

5g) le domaine publlc de l"Interfluve Gatineau-Llèvre, solt essentlellement le territoire environnant les lacs des Trente et Un Mille et Polsson-Blanc:

5h) le domaine public Ouareau, dont la forét Ouareau;

5I) le domaine public de la Haute Gatineau, dont le réservolr Baskatong.

Ces grands domaines publics sont surtout connus des amateurs de grande nature, chasseurs, pêcheurs, randonneurs, canoteurs, campeurs. Ils sont tous sltués en pérlphérle nord des territolres développés et municipallsés des Laurentides, excepté les domaines publics Gatineau-Llève el PapineauLabelle, qui constutuent deux îlots de grande nature au coeur de la réglon.

Lá villéglature y est relativement peu développée et consiste surtout en des chalets ou bâtiments d"hébergement hérités des clubs privés de chasse et pêche par les pourvoiries. ZEC et les réserves faunlques gérées par le ministère de l'Environnement et de la Faune. Bien que l'exploltation forestlère ait contribué à diminuer la qualité de la forêt orlglnale, le caractère grande nature et sauvagerie de ces grands espaces est relativement bien conservé. Un réseau très élaboré de chemins forestlers permet d'accéder à tous les lacs et cours d'eau et falt le bonheur des propriétaires de VTT et de véhlcules $4 \times 4$. Ces grands espaces de grande nature sont parml les plus utilisés au Ouébec en ralson de leur proximité des grands bassins de population.

$6^{*}$ Les espaces à statut particulier, solt les territoires juridiquement délimités et affectés à des usages spéciflques

Ce sont:

6a) le Parc de la Gatineau, situé sur la bordure des Laurentides au nord-ouest de Hull et adminlstré par la Commission de la capitale nationale pour des fins de récréation el de conservation:

6b) le Parc du Mont-Tremblant, vaste territolre chevauchant les massifs montagneux les plus importants des Laurentides et administré par le Ministère de I'Environnement et de la Faune pour des fins de récréation et de conservation:

6c) la Réserve écologlque des Laurentides, sltuéce à SaintFaustin, pour protéger pour les générations futures, un écosystème de la forêt des Laurentides; 6d) la Réserve Indlenne de Manlwakl, territolre sur la rlvıère Désert à l'usage exclusif des membres de la bande;

6e) la réserve indienne de Doncaster, territoine forestler et montagneux, à l'est de Sainte-Lucle et à la tête des eaux de la rivière Doncaster, à l'usage exclusif des autochtones autorisés:

60) la réserve de la Petite-Nation, grand domalne privé, à l'usage récréotouristıque de lá cllentèle du Château Montebello.

Parml ces espaces, seuls les parcs de la Gatineau et du Mont-Tremblant sont connus du grand public et appropriés à des fins récréotouristıques. Le parc du Mont-Tremblant attire une nombreuse cllentèle et compte, grâce à la station tourlstlque développée à sa périphérle méridlonale, l'attrait princlpal des Laurentides. Le parc de la Gatineau, à cause de son positionnement comme un des attraits de la réglon de la capltale fédérale, accuellle de nombreux visiteurs en provenance de toutes les partles du Canada et de l"étranger. C'est. le seul endroit au Québec oû on trouve un parkway, c'est-âdire une route-promenade aménagée spéciflquement pour les plaisirs de la randonnée automobile. La réserve de la PetiteNation est un vaste domalne foncler privé peu connu et situé à l'écart des voles de communication. Quant à la réserve écologique des Laurentldes, elle est accessible aux seuls scientifiques autorlsés à y séjourner par le ministre.

$7^{*}$ Entité spatlale au caractère particuller, le couloir Nolre-Sauvage-Matawin, solt la vallée profonde et encalssée qul permet de franchlr les hautes Laurentldes entre Salnte-Émllie-de-l'Énergle et SalntMlichel-des-Saints

Ce coulolr étrolt est le seul passage possible à travers les massifs montagneux importants qul barrent le passage vers le bassin du Saint-Maurice. La route 131 qul l'emprunte est encadrée par des hautes parols abruptes jusqu'au col qul marque la limite de la ligne de partage des eaux du bassin du Saint-Maurice. C'est un des rares endroits des Laurentides présentant des caractéristiques de paysages de haute montagne. Au nord du col, la route 131 débouche dans la vallée de la rivlère Sauvage, belle auge glaclalre où champs et páturages rappellent la colonlsation agricole du début du siècle et oì les panoramas sont parmI les plus beaux des Laurentides. Quant à la vallếe de la Matawin, elle est surtout connue pour Salnt-Michel-des-Saints et le réservolr Taureau, hauts-lieux du tourisme de grande nature, au nord de Lanaudlère.

\section{Conclusion}

Ce bref portrait des Laurentides brossế à grand tralt révèle une organisation spatiale du territolre beaucoup plus complexe que celle reflétée dans la littérature de promotion touristlque nouvelle vague où le visiteur est invité à venir davantage consommer un produit touristique quà découvrir un espace ou un coln de pays. Il est à souhaiter que l'approche géographique puisse être introdulte davantage dans la promotion touristique des Laurentides, et qu"on y amène les visiteurs à sortir des sentiers battus et plus préclsément des roules 105, 117 et 125 . Nous espérons que cette brève présentation des Lá rentides du nord de Montréal et d'Ottawa va en permettre une meilleure compréhension et 
surtout demontrer la relation complexe de leur mise en valeur et de leur organisation spatiale. Parml les quelque trente entités spatlales différentes délimilées et brièvement décrites dans cet article, environ quatre ou cinq concentrent la majorité des installations touristiques et accaparent la part princlpale du marché des visiteurs des Laurentides du nord de Montréal et d'Ottawa. Les quelque vingt-cinq autres entités spatlales n'en sont pas moins intéressants à visiter, mals elles sont ignorées du public parce que méconnues el situées à l'extérieur des corridors principaux d'accès aux Laurentides.

\section{Les Laurentides du Nord de Montréal el d'Ottawa: un aperçu de l'organisation spatiale}

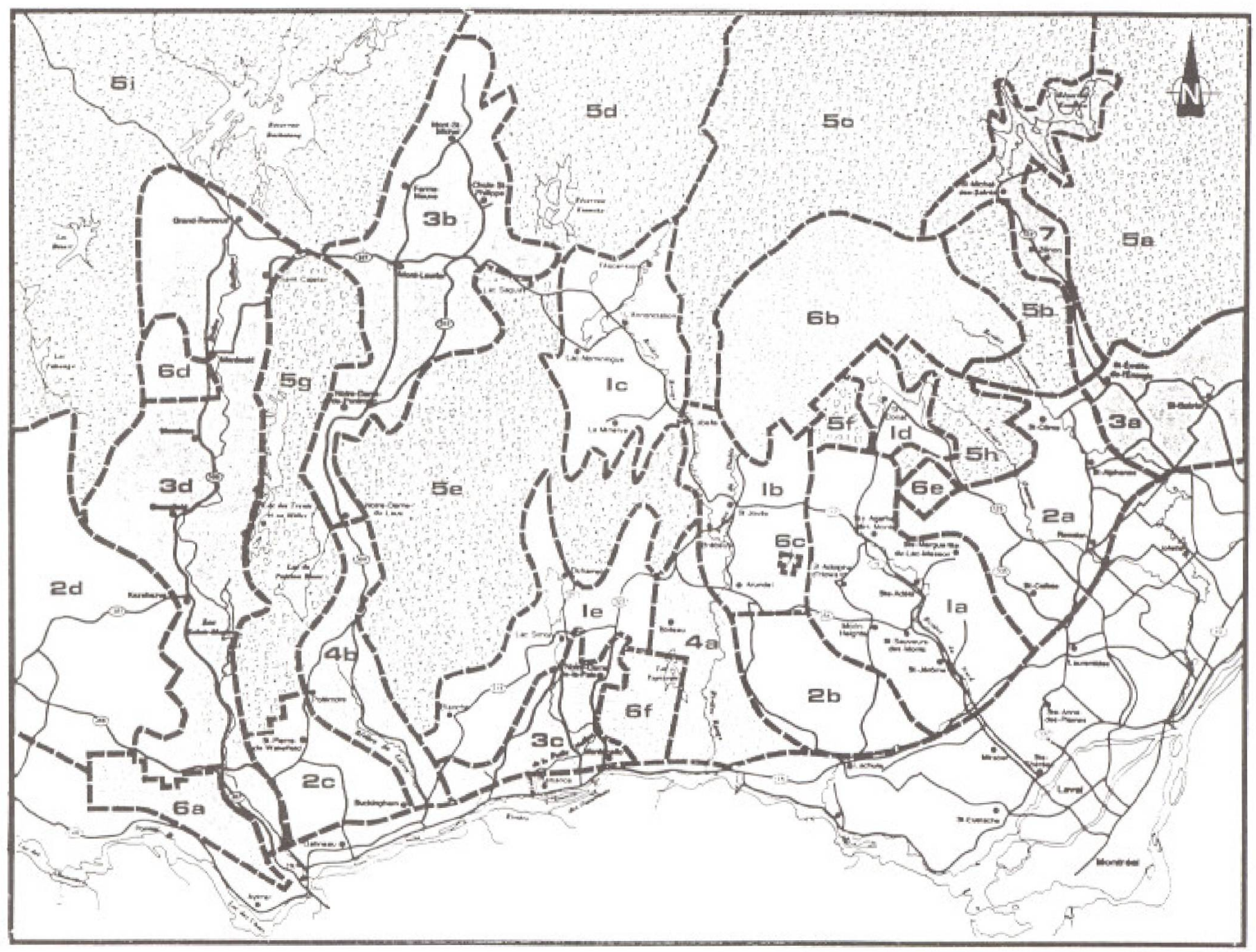

1 Les espaces de villéglature et de récréotourisme structurés par une route nationale ou réglonale

2 Les espaces de villéglature de la bordure méridionale des Laurentides

3 Les espaces agricoles et de villéglature

4 Les espaces aux paysages agraires remarquables

5 Les espaces de grande nature axés sur l'exploitation contrôléc de la faune et de la forêt

6 Les espaces à statut particulier (parc et réserve écologique: réserve amérindienne)

7 Le couloir Nolre-Sauvage-Matawin 